CARDIOVASCULAR MEDICINE

\title{
Does the use of a syncope diagnostic protocol improve the investigation and management of syncope?
}

\author{
D J Farwell, A N Sulke
}

Heart 2004:90:52-58

See end of article for authors' affiliations .....................

Correspondence to:

Dr A N Sulke, Department of Cardiology, Eastbourne District General Hospital, King's Drive, Eastbourne, East Sussex, UK; djfarwell@bigfoot.com

Accepted 22 May 2003
Objectives: To assess the efficacy of a protocol designed to improve the diagnosis and management of syncope.

Design: Prospective outcome analysis of all patients presenting with syncope for the 12 month period from 1 November 2000 to 31 October 2001, compared with a retrospective study of all patients presenting with syncope during the calendar year 1998. Use of the protocol commenced in September 2000.

Setting: Eastbourne District General Hospital, serving a population of approximately 250 000; $25 \%$ are older than 65 years.

Subjects: 421 consecutive patients presenting with syncope, investigated prospectively in January 2000 and compared with 660 patients retrospectively analysed for the calendar year 1998.

Results: In 1998, 71\% of patients with syncope received a diagnostic classification. In January 2000 there was an appropriate diagnostic hypothesis for every patient. Ultimately a diagnosis was made for $78 \%$ of patients according to accepted criteria $(p=0.003)$. Use of tests with the highest diagnostic effectiveness, such as tilt tests, increased in 2001 and many tests were used more appropriately (such as echocardiography). However, non-diagnostic tests were still frequently used (such as chest radiography, electroencephalography, and carotid Doppler studies). Costs of investigation and hospital stay rose from $£ 611$ to $£ 1384$ ( $€ 874$ to $€ 1980$ ) per patient $(p<0.001$ ), with cost per diagnosis increasing from $£ 870$ $(€ 1245)$ in 1998 to $£ 1949$ (€2790) ( $<<0.001)$.

Conclusion: The syncope protocol improved diagnosis and the use of appropriate investigations. However, significant inappropriate investigation and hospital admission still occurred. The protocol allowed reliable triage of syncopal patients into high and low risk groups.
$\mathrm{S}$ yncope is a common disorder with an annual incidence of between 1.3 and 2.7 episodes per thousand of population per annum. ${ }^{12}$ Retrospective studies suggest that up to $40 \%$ of the general population have experienced an episode of syncope in their lifetime. ${ }^{3}$ Syncope is the cause of $6 \%$ of all acute medical admissions and of $3 \%$ of accident and emergency department consultations. ${ }^{56}$ It therefore places a major burden on health care budgets. The causes of syncope are diverse and clinicians use a wide range of investigations to try to achieve a diagnosis.

A retrospective study reviewed every patient presenting to Eastbourne Hospital from 1 January to 31 December 1998 with an episode of syncope. ${ }^{7}$ This investigation showed that among the 660 patients presenting to our institution with confirmed syncope, a diagnosis was not reached for $29 \%$. Investigations were not targeted at those at high risk from recurrent syncope or with life threatening causes of syncope.

Other investigators have also shown that the management of syncopal patients presenting to the accident and emergency department or acute medical team is often suboptimal. ${ }^{8-10}$ However, the use of a diagnostic protocol has been shown to improve the management of such patients. ${ }^{11}{ }^{12}$ The purpose of our study was to examine the clinical and financial impact of such a protocol on the management of patients with syncope presenting on an emergency basis to our institution.

\section{METHODS}

Eastbourne District General Hospital is a busy non-tertiary centre on the south east coast of England. It serves a population of approximately $250000,24 \%$ of whom are older than 65. With the support of the Eastbourne medical directorate and after an extensive literature review, a simple syncope diagnostic and management protocol was designed to risk stratify syncopal patients and to ensure that appropriate and cost effective investigations were conducted as soon as possible after initial presentation. The protocol was then used to guide the management of all syncopal patients presenting to the Eastbourne District General Hospital over the study period. Following a two month run-in phase, data were collected prospectively for one year. Patients were identified by medical and nursing staff from every hospital department. The investigators reviewed each patient individually. Data were collected regarding length of hospital stay, investigations performed, and diagnosis reached. Patients were followed up for at least six months after their initial presentation. Results after changes to practice were compared with the findings of the retrospective audit of all 1998 admissions for syncope. $^{7}$

The presumed diagnosis was assigned by the investigators after clinician initiated diagnostic procedures. The hypothesised diagnoses made by junior and consultant clinicians were documented and compared.

\section{Definitions}

Terms were defined as follows:

- Syncope-Transient loss of consciousness and postural tone $^{13}$

Abbreviations: CSM, carotid sinus massage; ELR, external loop recorder; ILR, implantable loop recorder 
- Postural hypotension-Decrease in systolic blood pressure of more than $50 \%$ from supine or to $<60 \mathrm{~mm} \mathrm{Hg}$ during five minutes in the upright position, associated with presyncope or syncope ${ }^{12} 14$

- Vasovagal syncope-Loss of consciousness induced by tilt testing in the presence of bradycardia, hypotension, or both, or when syncope occurred in the absence of cardiac disease and with appropriate clinical features ${ }^{15-19}$

- Carotid sinus syncope-Syncope induced by carotid sinus massage (CSM), performed in the upright position, in the presence of bradycardia, hypotension, or both ${ }^{17}$

- Drug induced syncope-Defined when a clear temporal relation between drug consumption and syncope could be proved

- Arrhythmic syncope-Diagnosis based on the following findings of standard ECG, 24 hour Holter monitoring, seven day event monitoring (external loop recorder (ELR)), or implantable loop recorder (ILR): any arrhythmia associated with syncope, ventricular pauses $>3 \mathrm{~s}$, Mobitz II heart block or third degree atrioventricular block while awake; and ventricular tachycardia. ${ }^{20}$

The syncope diagnostic and management protocols are shown in fig 1.

\section{Phase 1}

If, after presentation with syncope, the history, physical examination (including lying and standing blood pressure), 12 lead ECG, full blood count, plasma glucose, urea, and electrolytes fail to lead to a diagnosis the patient entered phase 2 .

\section{Phase 2}

Hypothesised diagnosis were as follows: cardiac syncope (any cardiac abnormality identified in phase 1); neurological syncope (focal neurological signs such as epilepsy, cerebrovascular accident, trauma, and neoplasia identified in phase 1); and vasovagal syncope (any form of syncope apart from the above).

The management protocol of patients falling in to each of the hypothesised diagnoses was as follows.

\section{Management of patients with cardiac syncope}

Patients with cardiac syncope were admitted for 24 hours of cardiac telemetry plus an echocardiogram if obstructive cardiac pathology was suspected.

If syncope was more frequent than once a week, a seven day patient activated ELR was used (R Test, Novocor, France).

If syncope was less frequent than once weekly or if ELR failed to capture the event, then a tilt test was performed (with CSM) and an ILR inserted (Reveal Plus, Medtronic, Minneapolis, Minnesota, USA) if indicated.

\section{Management of patients with neurological syncope}

Serum sodium, calcium, and magnesium concentrations were measured. If a patient was taking anticonvulsants then serum concentrations of the drug were also measured. Brain imaging was considered and other investigations performed as indicated. Appropriate follow up or admission, if considered necessary, were arranged.

\section{Management of patients with vasovagal syncope}

Patients were reassured. Admission was unnecessary unless otherwise indicated. If the patient had more than one episode, then a tilt test was performed (with CSM) and an ILR implanted if indicated.

\section{Patient follow up}

The investigators reviewed all patients with recurrent syncope. All other patients had a case note review. Study census date was 1 April 2002.

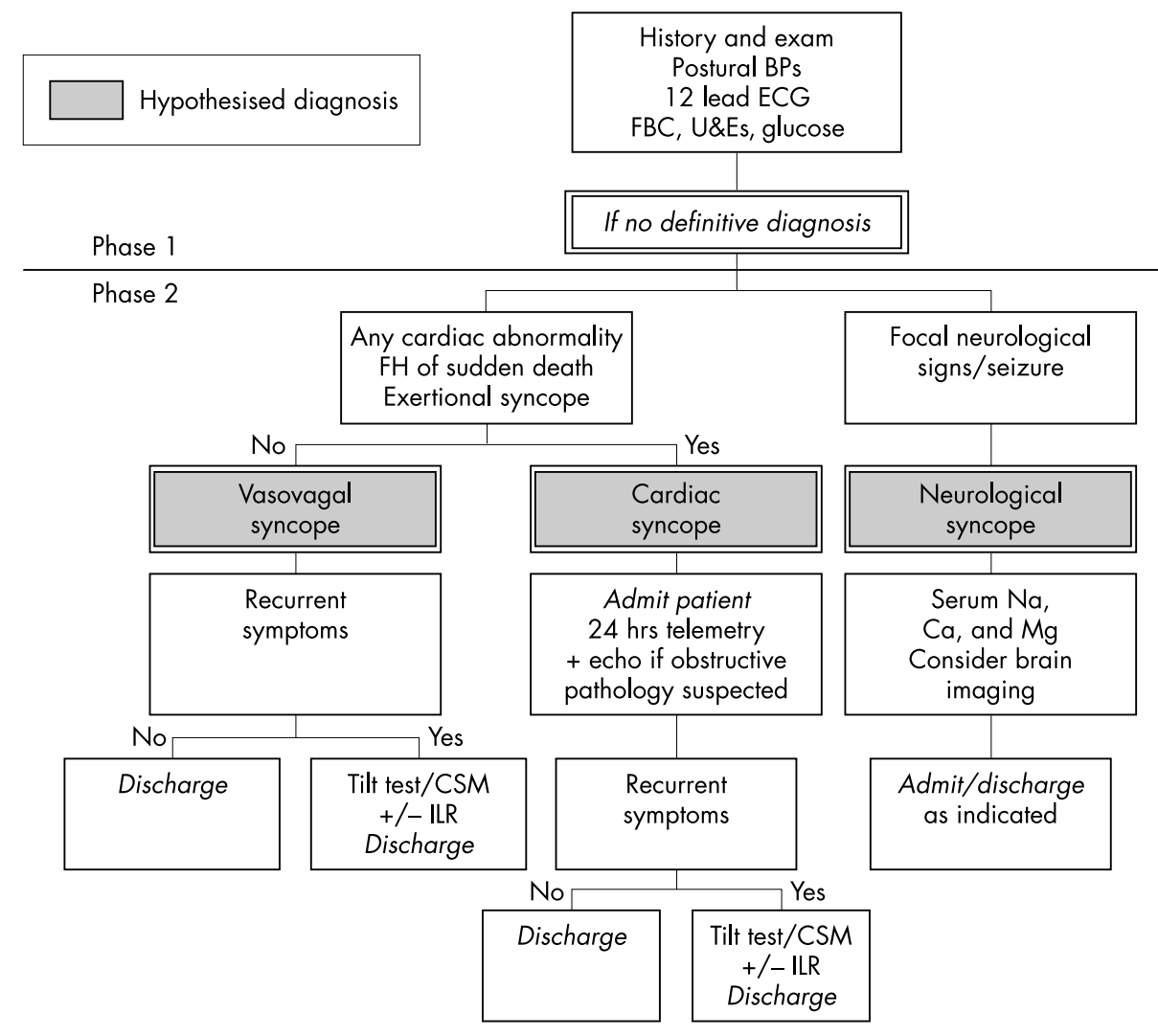

Figure 1 Protocol flow chart 


\section{Statistical analysis}

Data were analysed with StatsDirect version 1.9.8. Comparative analysis was by the use of unpaired Student's $t$ test, Mann-Whitney U test, $\chi^{2}$ test, and Fisher's exact test, where appropriate. Data are displayed as mean (SD) with median quoted where the data are non-parametric. A probability value of $\mathrm{p}<0.05$ was considered significant.

\section{RESULTS}

Twenty eight $(4.2 \%)$ of the 660 patients who presented in 1998 also presented in January 2000 and were excluded from the 2000/1 data, leaving 421 patients who presented with syncope solely during the study period January 2000. The mean (SD) age was 71 (18) years and 54\% were women. The median number of syncopal episodes per patient was two (1-4 interquartile range) and the median duration of symptoms was four months (0-24 interquartile range). The diagnosis was made for 22 patients (5.2\%) after phase 1 (fig $2 \mathrm{~A}$ ). A diagnostic hypothesis with which to guide further management was then made for all patients (fig $2 \mathrm{~B}$ ). Figure $2 \mathrm{C}$ summarises the presumed diagnoses.

\section{Diagnostic hypothesis vasovagal syncope}

A vasovagal syncope diagnostic hypothesis was made for 137 patients (34\%). Of this group, 30\% were admitted to hospital, against protocol recommendation. At the end of follow up all patients with a vasovagal diagnostic hypothesis had a presumed diagnosis. A non-vasovagal bradycardia diagnosis was presumed for two (1.5\%) patients with a vasovagal diagnostic hypothesis and they received a cardiac pacemaker. Of the remainder, the cause of syncope was psychogenic for two $(1.5 \%)$ patients, epilepsy was diagnosed for one $(0.7 \%)$, and the type of syncope was vasovagal in the rest (96\%) (fig 3).

\section{Diagnostic hypothesis cardiac syncope}

The diagnostic hypothesis was cardiac syncope for 195 patients (49\%), of whom 73\% (142/195) were admitted to hospital (the protocol recommends that all patients with cardiac syncope be admitted). A diagnosis was presumed for $53 \%$ of this group of patients at the end of follow up. Vasovagal syncope was diagnosed for 60 of these patients (31\%). Arrhythmic syncope was diagnosed for 32 patients ( $16 \%)$. Six patients (3\%) had significant aortic stenosis (fig 3 ).

\section{Diagnostic hypothesis neurological syncope}

A neurological diagnostic hypothesis was made for 67 (17\%) patients, of whom 95\% (64/67) were admitted to hospital (no protocol recommendation). At the end of follow up a presumed diagnosis had been made for all patients with a neurological diagnostic hypothesis. A cerebrovascular accident was diagnosed for $35(52 \%)$ patients. Fifteen $(22 \%)$ patients had an isolated seizure and epilepsy was diagnosed for $15(22 \%)$ patients. Two (3\%) patients had a psychogenic cause of syncope (fig 3).

\section{Protocol sensitivity and specificity}

A central nervous system related cause of syncope was the presumed diagnosis for 65 of the 67 patients with a hypothesised diagnosis of neurological syncope, making the process $98 \%$ specific. A type of vasovagal syncope was the presumed diagnosis for 132 of 137 patients with a vasovagal hypothesised diagnosis, making the process $96 \%$ specific. Of the 195 patients with a cardiac hypothesised diagnosis no diagnosis was made for 93 (48\%). For patients with a presumed cardiac diagnosis the protocol specificity was 39\% and sensitivity was $95 \%$.

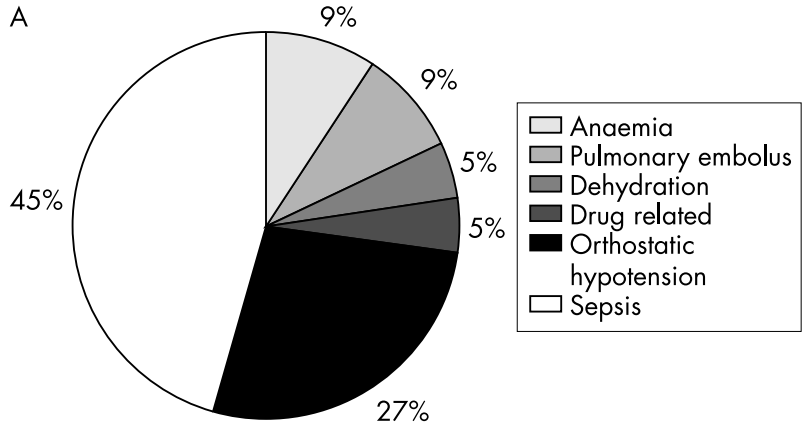

B
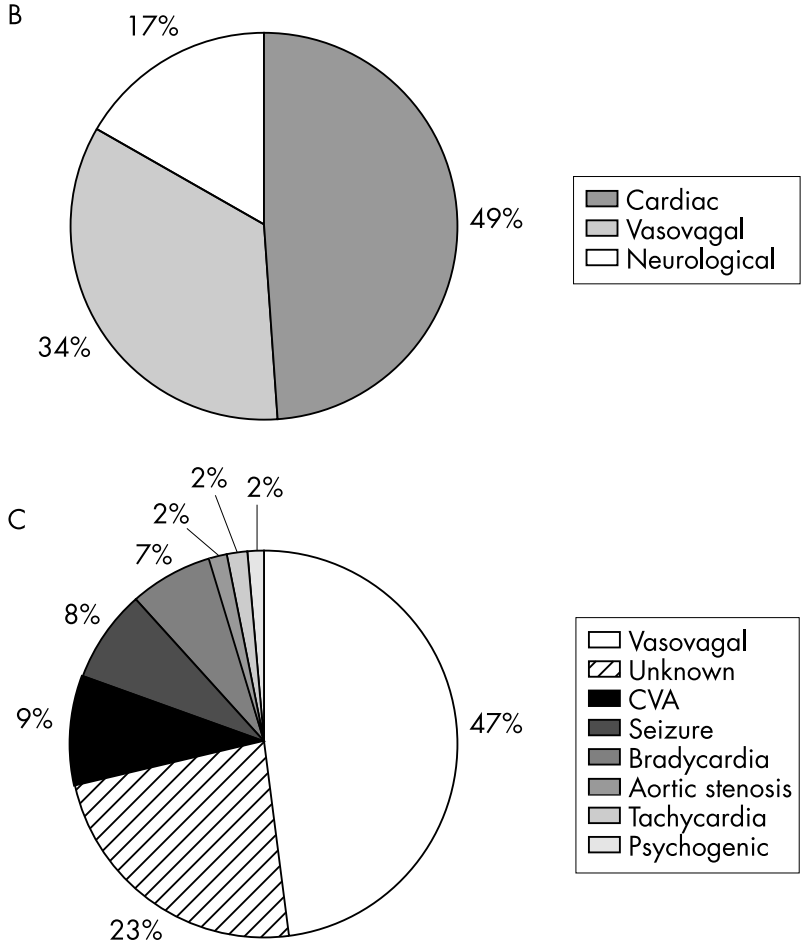

Figure 2 (A) 2001 phase 1: diagnoses (22 cases). (B) 2001 phase 2: diagnostic hypotheses. (C) $2000 / 1$ phase 2: presumed diagnosis 399 patients CVA, cerebrovascular accident.

\section{Resources used}

Hospital stay

Mean (SD) length of stay was 4.4 (7.5) days (median two days). Hospital stay was prolonged for patients with neurological syncope (10.2 (4.4) days) and for patients requiring social services (mean wait for social input was 1.7 (4.9) days). If the protocol had been complied with, the 421 patients would have required 300 bed days. The actual occupancy was 1852 days.

\section{Phase 1 tests}

All patients underwent at least one 12 lead ECG recording (average 1.30) and had an adequate history and examination undertaken. Blood tests, required by the protocol, were done for $79 \%$ of patients (mean (SD) 1.7 (1.9)) (table 1). There were no significant differences in the number of blood tests and ECGs performed between the 1998 and January 2000 studies or in the number diagnoses achieved (table 1).

History and examination resulted in a diagnosis for 166 patients in January 2000 with a diagnostic effectiveness of 2.5. This was the second most effective diagnostic modality 


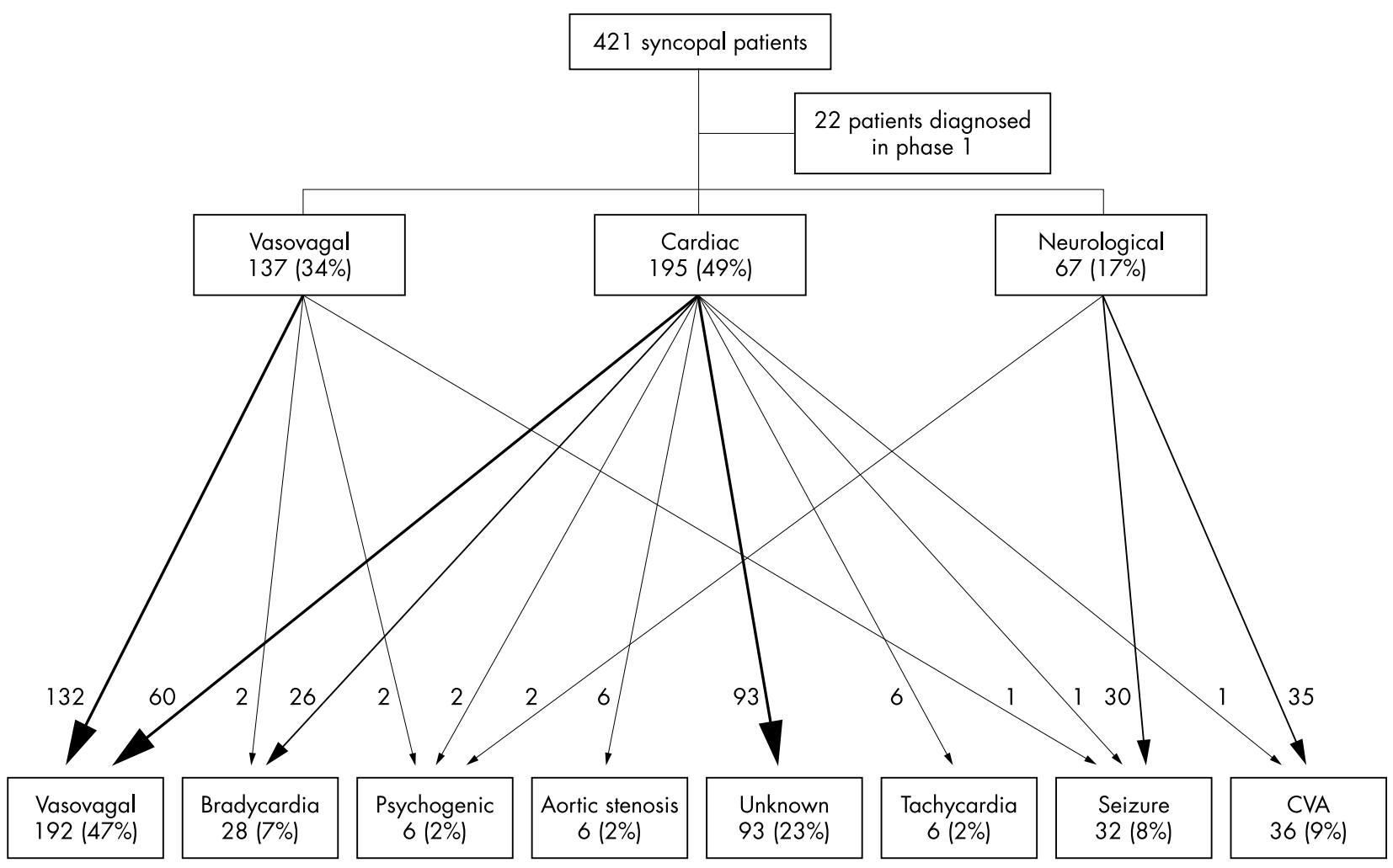

Figure 3 Diagnostic outcome of all patients presenting 2000/1. Percentages shown for definitive diagnoses exclude the initial 22 patients diagnosed in phase 1.

after tilt testing in both 1998 (244 diagnoses, diagnostic effectiveness 2.67) and January 2000 (table 1).

\section{Cardiac investigations}

Every patient with cardiac syncope diagnostic hypothesis underwent investigation by 24 hour Holter monitor. However, $39(58 \%)$ patients in the neurological category and $116(85 \%)$ in the vasovagal category also underwent investigation by 24 hour Holter monitor, against protocol recommendations. This practice resulted in only one diagnosis among the 155 Holter investigations for patients not in the cardiac syncope category.

Among patients with cardiac syncope diagnosed during phase 1, Holter monitoring was diagnostic in 18 of 195 investigations (one diagnosis in 10.8 investigations). Thus, Holter monitoring was 16 times (95\% confidence interval 2.20 to $600, \mathrm{p}<0.001$ ) more likely to be diagnostic for these patients. More patients in January 2000 underwent investigation by 24 hour Holter monitoring than in 1998 (83\% v $34 \%, \mathrm{p}<0.001)$. The number of Holter investigations required to make a diagnosis rose from 9.8 in 1998 to 18.4 in January 2000 ( $\mathrm{p}=0.048)$.

A greater proportion of patients received ELRs in January $2000(10 \%$ v 3\%). However, diagnostic rates fell: 2.6 ELRs were required to make a diagnosis in 1998 compared with 20 in January $2000(\mathrm{p}<0.001)$. An ILR was implanted in $25 \%$ of patients in January 2000. None were implanted in 1998. ILRs lead to a diagnosis for 30 patients by census date $(29 \%$

\begin{tabular}{|c|c|c|c|c|c|c|}
\hline \multirow[b]{2}{*}{ Tests } & \multicolumn{3}{|c|}{ Number of tests performed } & \multicolumn{3}{|c|}{$\begin{array}{l}\text { Diagnostic effectiveness } \\
\text { (tests/diagnosis) }\end{array}$} \\
\hline & 1998 & $\begin{array}{l}\text { January } \\
2000\end{array}$ & $\mathrm{p}$ Value & 1998 & $\begin{array}{l}\text { January } \\
2000\end{array}$ & p Value \\
\hline Patients & 660 & 421 & & & & \\
\hline History and examination & 660 (100\%) & 421 (100\%) & NS & 2.68 & 2.5 & NS \\
\hline Holter monitoring & $226(34 \%)$ & $350(83 \%)$ & $<0.001$ & 9.8 & 18.4 & 0.48 \\
\hline ELR & $26(3 \%)$ & $40(10 \%)$ & $<0.001$ & 2.6 & 20 & $<0.001$ \\
\hline ILR & 0 & $103(25 \%)$ & & 0 & 3.5 & \\
\hline Brain imaging & $87(13 \%)$ & $20(5 \%)$ & $<0.001$ & 87 & ND & NS \\
\hline Blood tests & $1058(160 \%)$ & 733 (174\%) & NS & 118 & 183 & NS \\
\hline ECG & $881(133 \%)$ & $547(130 \%)$ & NS & 147 & 547 & NS \\
\hline Echocardiography & $64(10 \%)$ & $62(15 \%)$ & 0.012 & 64 & 10 & 0.05 \\
\hline Tilt table test & $24(4 \%)$ & $214(51 \%)$ & $<0.001$ & 2.67 & 2.14 & NS \\
\hline Chest radiography & $193(29 \%)$ & $206(49 \%)$ & $<0.001$ & ND & ND & \\
\hline EEG & $26(4 \%)$ & $11(3 \%)$ & NS & ND & ND & \\
\hline Carotid Doppler study & $10(2 \%)$ & $14(3 \%)$ & NS & ND & ND & \\
\hline
\end{tabular}

EEG, electroencephalography; ELR, external loop recorder; ILR, implantable loop recorder; ND, non-diagnostic. 
of those who received one). It was the third most effective investigation in January 2000.

There was a ninefold increase in the number of tilt tests performed in January 2000 over 1998 (214 v 24, p < 0.001). The number of tilt tests required to make a diagnosis fell slightly from 2.67 to 2.14 (not significant; table 1), suggesting that this test was appropriately used as directed by the protocol. Tilt tests had the highest diagnostic effectiveness in both 1998 and January 2000.

Use of echocardiograms increased in January 2000 (15\% v $10 \%, \mathrm{p}=0.012$ ) and $75 \%$ were performed because of significant clinical findings (for example, murmur of aortic stenosis). Echocardiograms performed in the absence of cardiac signs failed to provide any useful diagnostic information. Ultimately six cases of syncope were attributed to aortic stenosis, all diagnosed by clinical findings and confirmed by echocardiography. The number of examinations required to make a diagnosis fell from 64 in 1998 to 12 in January $2000(p=0.05)$. This suggests improved use of this investigation with good protocol compliance.

\section{Other investigations}

Chest radiographs were done for $49 \%$ of patients, an increase from $29 \%$ in 1998 ( $p<0.001$ ), against protocol recommendations. In the absence of significant clinical findings (two patients with pneumonia), these radiographs provided no additional diagnostic information and did not affect the management of any patient.

Similarly, in the absence of focal neurological signs brain imaging provided no useful diagnostic information in any patient. In January 2000 brain imaging did not alter a clinical diagnosis in any patient. However, there was a significant reduction in the number of such investigations from 1998 ( $13 \%$ in $1998 \vee 5 \%$ in $2000, p<0.001$ ) based on protocol recommendations.

There were no significant differences in the rate of investigation by electroencephalography or carotid Doppler studies between 1998 and January 2000. Neither investigation resulted in a diagnosis in any patient (table 1 ).

\section{Costs of diagnosis}

Costs arising from hospitalisation rose by 49\% (£299 200 to $£ 448200$ ) due to increased hospital stay. On average $40 \%$ of hospital stay was spent awaiting a social referral in January 2000. Investigational costs also rose from 1998 to January 2000 (table 2). Overall, total costs more than doubled from 1998 to 2000 (table 2). ILRs were not used in 1998, so two cost comparisons were undertaken, one with ILR costs included and one without. Investigational costs rose both with and without ILRs. This was partially offset by higher diagnostic rates; however, cost per diagnosis rose from $£ 870$ to $£ 1949$ excluding ILR ( $p<0.001$ ), or $£ 2114$ with ILR.

\section{DISCUSSION}

Syncope in the general population has a relatively benign prognosis. ${ }^{2141819}$ However, syncope secondary to a cardiac cause carries a one year mortality of 18$33 \% .{ }^{6}{ }^{11}$ Furthermore, patients suffering from recurrent syncope have a greatly reduced quality of life (similar to those with severe rheumatoid arthritis or chronic low back pain). ${ }^{21}$

This study has shown that it is possible to improve the diagnosis of syncope in a district general hospital by the use of a simple diagnostic protocol, which focuses management on clinical judgement and specific and relevant clinical investigations. Our protocol flow chart was drawn up before the publication of the European Society of Cardiology guidelines but is similar to the flow chart to be found within them. We did not refer any patients for electrophysiological studies and a good diagnostic yield was achieved without their use. In part this resulted from the use of the ILR as a substitute. This reduced reliance on electrophysiological studies reflects European practice and is encouraged by the British guidelines for the use of implantable cardioverterdefibrillators. ${ }^{22}$

Data from 421 consecutive patients with syncope were prospectively compared with the 660 patients admitted in 1998. This is within the range observed in the Framingham study ${ }^{11}$ (325-675 cases of syncope expected per annum for our catchment population). The population studied is slightly older than that previously studied but our local population is older than usual (25\% older than 65$).^{813}$

The use of the protocol led to a decline in the number of undiagnosed cases of syncope from 29\% in 1998 to $0 \%$ without a hypothesised diagnosis in January 2000. Furthermore, a presumed diagnosis was made in $78 \%$ of all cases, allowing appropriate patient counselling and evidence based treatment to be instituted, significantly better than in 1998. Similar improvements have been noted in the Italian OESIL (osservatorio epidemiologico della sincope nel Lazio) study, which recorded a reduction in uncategorised syncope discharges from $54.4 \%$ before to $17.5 \%$ after a protocol was applied. ${ }^{11}$

The protocol is specific, allowing triage of patients into lower risk groups (such as neurological and vasovagal) who may be investigated as outpatients, thus reducing costs without risking patient safety. One year mortality of patients with cardiac syncope $(18-30 \%)$ is consistently higher than of patients with a non-cardiac cause $(0-12 \%) .^{6}{ }^{14} 23-25$ Importantly it sensitively triages those who have a cardiac cause of syncope and are at high risk of death.

\section{Protocol compliance}

The syncope protocol appears to have failed in its secondary purpose, to reduce hospitalisation and to reduce costs. Failure of compliance is often thought to be caused by a combination of poor communication and education. ${ }^{26}$ Protocol compliance is often as low as $30 \%,{ }^{27}$ although it was partial noncompliance in our study, which is difficult to quantify. Extended hospital stay and high initial admission rates were often due to elderly patients requiring social support.

\begin{tabular}{|c|c|c|c|}
\hline & $1998^{8}$ & January 2000 (with ILR) & January 2000 (without ILR) \\
\hline $\begin{array}{l}\text { Investigational costs (£) } \\
\text { Cost for nights in hospital (£) } \\
\text { Total costs (£) } \\
\text { Cost/patient (£) } \\
\text { Cost/diagnosis (£) }\end{array}$ & $\begin{array}{l}104285 \\
299200 \\
403485 \\
611(560)^{*} \dagger \\
870(683)^{*} \dagger\end{array}$ & $\begin{array}{l}237392 \\
448200 \\
685529 \\
1628(1432)^{*} \\
2114(1857)^{*}\end{array}$ & $\begin{array}{l}134392 \\
448200 \\
582592 \\
1384(1215) \dagger \\
1949(1698) \dagger\end{array}$ \\
\hline
\end{tabular}


This was not accommodated within the remit of the protocol.

The number of brain imaging investigations was significantly reduced and, although the number of echocardiograms performed increased, they appeared to be better targeted. There was increased and more appropriate use of ELRs, ILRs, and tilt tests as directed by the protocol, but 24 hour Holter monitoring was overused and poorly targeted. With improved protocol compliance the cost of Holter monitoring would have been halved if it had been restricted to patients with a cardiac diagnostic hypothesis only ( $£ 35000$ to $£ 18000$ ). Holter cost per diagnosis would have fallen from $£ 1220$ to $£ 642$ with a $<5 \%$ fall in sensitivity.

There was no significant change in the rates of investigation by blood tests, ECG, electroencephalography, or carotid Doppler echocardiography. Thus, it appears that clinicians are keen to perform additional investigations but are reluctant to dispense with their usual investigations. Serial cardiac enzyme analysis was the principal cause of repeated blood testing and this led to unnecessary hospitalisation. No myocardial infarction was diagnosed in any of the 421 patients studied. The low yield of this type of investigation in syncopal patients has also been noted by other investigators. ${ }^{28}$

Total hospital stay should have been 300 nights if the protocol had been strictly adhered to. Partly because of lack of compliance, hospital stay was six times longer. The causes for prolonged hospital stay were multiple. In January 2000 the major cause for delayed discharge was inadequate social work review and placement. Forty per cent of hospital stay for syncopal patients was spent waiting for social referral and placement.

\section{Investigational costs}

ILRs were not used in 1998. Each device cost $£ 1350$ and accounted for $43 \%$ of the incurred investigational costs in January 2000. However, the ILR had a high diagnostic rate (three devices per diagnosis in our population). This compares with other studies in which diagnostic rates varied from $29-69 \% .^{25}{ }^{29}$

We found an increase in investigational costs both with and without ILRs. Previous studies have suggested that a more structured approach to diagnosing syncope along with the use of ILRs can lead to a reduction in syncope costs. ${ }^{30}$ However, these studies had highly selected populations of patients who had already incurred costs generated from extensive previous investigations.

Many improvements in health care have led to greater financial costs. Improvements in outcome must be balanced with an increased fiscal burden. ${ }^{31}$

\section{Study limitations}

Diagnoses from the retrospective 1998 analysis were made by attending clinicians and were corroborated only by case note review by the investigators, unlike those in the January 2000 prospective study, when all patients were clinically reviewed and followed up.

\section{Conclusions}

The study showed that a simple protocol for the investigation and management of syncope improves the diagnosis of the condition and consequently its management. It was especially successful in triaging patients at low and high risk of syncope.

Investigations should be targeted specifically on the basis of history, examination, and ECG. Tilt testing with CSM can be particularly useful in patients with normal hearts and in those with abnormal hearts and a normal 24 hour Holter result.
However, since the introduction of the protocol, the costs of diagnosing syncope have increased. This may have been caused by significant lack of adherence to some aspects of the protocol. There was no reduction in hospitalisation and many unnecessary and low yield investigations were still undertaken.

\section{ACKNOWLEDGEMENTS}

We thank Dr R Canavan for his help with data collection, Prof N Freemantle for statistical advice, and the clinicians of all departments of the Eastbourne District General Hospital, especially those of the medical directorate and A\&E departments.

\section{Authors' affiliations}

D J Farwell, A N Sulke, Department of Cardiology, Eastbourne District General Hospital, East Sussex, UK

\section{REFERENCES}

1 Savage DD, Corwin L, McGee DL, et al. Epidemiologic features of isolated syncope: the Framingham Study. Stroke 1985;16:626-9.

2 Chen L, Chen MH, Larson MG, et al. Risk factors for syncope in a communitybased sample (the Framingham Heart Study). Am J Cardiol 2000;85: 1189-93.

3 Murdoch BD. Loss of consciousness in healthy South African men: incidence, causes and relationship to EEG abnormality. S Afr Med J 1980;57:771-4.

4 Williams RL, Allen PD. Loss of consciousness. Aerosp Med 1962;33:545-51

5 Gendelman HE, Linzer M, Gabelman M, et al. Syncope in a general hospital patient population: usefulness of the radionuclide brain scan, electroencephalogram, and 24-hour Holter monitor. N Y State J Med 1983;83:1161-5

6 Kapoor WN. Evaluation and outcome of patients with syncope. Medicine (Baltimore) 1990;69:160-75.

7 Farwell D, Sulke N. How do we diagnose syncope? J Cardiovasc Electrophysiol 2002;13:S9-13.

8 Ammirati F, Colivicchi F, Minardi G, et al. [The management of syncope in the hospital: the OESIL study (osservatorio epidemiologico della sincope nel Lazio)]. G Ital Cardiol 1999;29:533-9.

9 Blanc JJ, L'Her C, Touiza A, et al. Prospective evaluation and outcome of patients admitted for syncope over a 1 year period. Eur Heart $J$ 2002;23:815-20.

10 Garcia CR, Sanjuan MR, Ruiz GR, et al. [Diagnostic accuracy of a protocol in the evaluation of unexplained syncope]. Rev Esp Cardiol 2001;54:425-30.

11 Ammirati F, Colivicchi F, Santini M. Diagnosing syncope in clinical practice: implementation of a simplified diagnostic algorithm in a multicentre prospective trial - the OESIL 2 study losservatorio epidemiologico della sincope nel Lazio). Eur Heart $J$ 2000;21:935-40

12 Oraii S, Maleki M, Minooii M, et al. Comparing two different protocols for tilt table testing: sublingual glyceryl trinitrate versus isoprenaline infusion. Heart 1999;81:603-5.

13 Soteriades ES, Evans JC, Larson MG, et al. Incidence and prognosis of syncope. N Engl J Med 2002;347:878-85.

14 Kapoor WN, Karpf M, Wieand S, et al. A prospective evaluation and follow-up of patients with syncope. N Engl J Med 1983;309:197-204.

15 Alboni P, Brignole M, Menozzi C, et al. Diagnostic value of history in patients with syncope with or without heart disease. J Am Coll Cardiol 2001;37:1921-8.

16 Natale A, Sra J, Akhtar M, et al. Use of sublingual nitroglycerin during headup tilt-table testing in patients $>60$ years of age. Am J Cardiol 1998;82:1210-3.

17 Kenny RA, O'Shea D, Parry SW. The Newcastle protocols for head-up tilt table testing in the diagnosis of vasovagal syncope, carotid sinus hypersensitivity, and related disorders. Heart 2000;83:564-9.

18 Eagle KA, Black HR, Cook EF, et al. Evaluation of prognostic classifications for patients with syncope. Am J Med 1985:79:455-60.

19 Manolis AS, Linzer M, Salem D, et al. Syncope: current diagnostic evaluation and management. Ann Intern Med 1990;1 12:850-63.

20 Brignole M, Alboni $P$, Benditt $D$, et al. Guidelines on management (diagnosis and treatment) of syncope. Eur Heart J 2001;22:1256-306.

21 Linzer M, Pontinen M, Gold DT, et al. Impairment of physical and psychosocial function in recurrent syncope. J Clin Epidemiol 1991;44:1037-43.

22 Lakehurst R, Carter Y, Berry C, et al. Guidance on the use of implantable cardioverter defibrillators for arrhythmias. London: National Institute for Clinical Excellence, 2000.

23 Silverstein MD, Singer DE, Mulley AG, et al. Patients with syncope admitted to medical intensive care units. JAMA 1982;248:1185-9.

24 Martin GJ, Adams SL, Martin HG et al. Prospective evaluation of syncope. Ann Emerg Med 1984;13:499-504. 
25 Krahn AD, Klein GJ, Fitzpatrick A, et al. Predicting the outcome of patients with unexplained syncope undergoing prolonged monitoring. Pacing Clin Electrophysiol 2002;25:37-41.

26 Lomas J. Words without action? Annu Rev Public Health 1991;12:41-65.

27 McPhee SJ. Performance of cancer screening. J Gen Intern Med 1986; 1:706-7.

28 Link MS, Laver EP, Homoud MK, et al. Low yield of rule-out myocardial infarction protocol in patients presenting with syncope. Am J Cardiol $2001 \cdot 88 \cdot 706-7$
29 Moya A, Brignole M, Menozzi C, et al. Mechanism of syncope in patients with isolated syncope and in patients with tilt-positive syncope. Circulation 2001;104:1261-7.

30 Krahn AD, Klein GJ, Yee R, et al. The high cost of syncope: cost implications of a new insertable loop recorder in the investigation of recurrent syncope. Am Heart J 1999;137:870-7.

31 Morgan JM. Cost-effectiveness of implantable cardioverter defibrillator therapy. J Cardiovasc Electrophysiol 2002:13:S114-7.

\section{IMAGES IN CARDIOLOGY}

\section{Pacemaker syndrome}

A

64 year old man presented with intermittent dizziness, palpitation, and dyspnoea. Three years ago, he underwent percutaneous coronary intervention with stenting for a mid right coronary artery stenosis and VVI pacemaker implantation for episodic symptomatic sinus pause. On admission, the 12 lead ECG showed a sinus rhythm with a rate of 54 beats/min (bpm). Two dimensional echocardiography revealed a dilated left ventricle with a global ejection fraction of $50 \%$ and mild mitral regurgitation. The VVI pacemaker was normal in pacing and sensing function, with pacemaker analysis and 24 hour Holter monitoring. Simultaneous recording of femoral arterial blood pressure and ECG revealed the arterial blood pressure dropped from 131/ 72 to $88 / 55 \mathrm{~mm} \mathrm{Hg}$ when VVI pacing rhythm appeared, as the sinus rate slowed below $60 \mathrm{bpm}$. During ventricular pacing rhythm, retrograde $\mathrm{p}$ waves were discernible after the
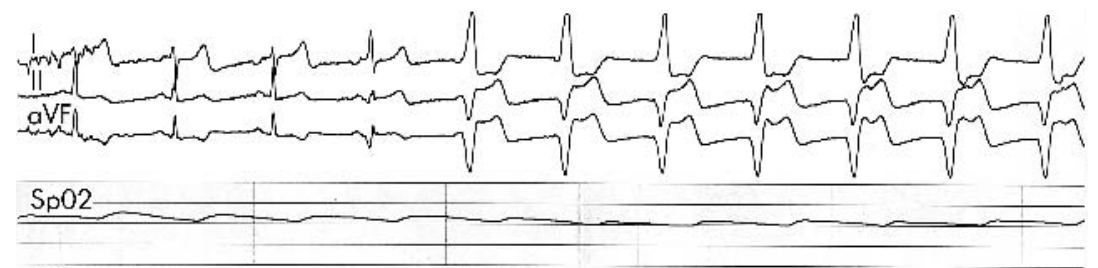

150

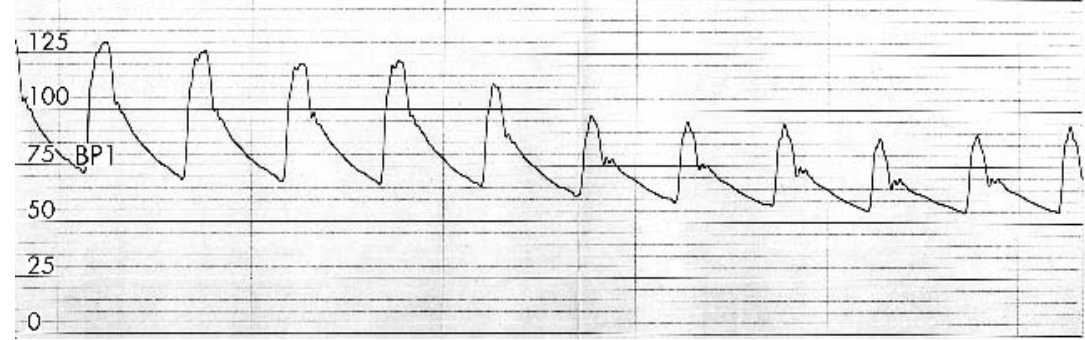
paced QRS complexes.

Y Ahn J G Cho cecilyk@hanmail.net

\section{ELECTRONIC PAGES}

\section{Heart Online case reports: www.heartjinl.com}

T he follow electronic only articles are published in conjunction with this issue of Heart.

\section{Rhabdomyolysis induced by a single dose of a statin} $S$ Jamil, P lqbal

Statins have been shown to cause myotoxicity and rhabdomyolysis. In most cases rhabdomyolysis occurs following the use of these drugs for at least one week. A case of rhabdomyolysis after just a single dose of simvastatin is reported. /e3

\section{Primary pericardial mesothelioma presenting as pericardial constriction: a case report \\ S Suman, P Schofield, S Large}

A 19 year old man presented with a six month history of chest pain, dyspnoea, and lethargy and was found on an echocardiogram to have a dilated left ventricle with a small pericardial effusion. Ramipril and a course of steroids were tried but serial echocardiograms showed a persistently thickened pericardium and slowly developing features of constriction. On computed tomography, a large mediastinal mass encasing the heart, along with para-aortic and paratracheal lymphadenopathy, was found. Right heart catheter studies showed equal pressures in all four chambers. His deteriorating clinical condition led to a pericardiectomy. Histology confirmed primary pericardial mesothelioma. The patient died soon after surgery.

(Heart 2004;90:e4) www.heartjnl.com/cgi/content/full/90/ 1/e3 